\title{
Synchronization of Chaotic Systems using Adaptive T-S Fuzzy Terminal Sliding-Function Control
}

\author{
Yun-Cheng Huang ${ }^{1}$ and Tzuu-Hseng S. $\mathrm{Li}^{2}$ \\ ${ }^{1}$ Department of Computer and Communication Engineering, National Kaohsiung \\ First University of Science and Technology, Kaohsiung, Taiwan, ROC, \\ ${ }^{2}$ Department of Electrical Engineering, National Cheng Kung University, \\ Tainan 70101, Taiwan, ROC \\ yjh@nkfust.edu.tw
}

\begin{abstract}
This paper presents an adaptive Takgi-Sugeno (T-S) fuzzy terminal sliding-function controller (AFTSFC) approach to synchronize two chaotic systems with parameter mismatch. First, an appropriate terminal sliding function (TSF) is designed and then represented by the $T$-S fuzzy model. The T-S fuzzy terminal sliding-function (FTSF) is applied to the control law. Different from classical terminal sliding mode control, which uses a discontinuous switching control law, the FTSF control uses a continuous control law and thus avoids the chattering problem. The linear matrix inequality (LMI) problem is solved to obtain the initial feedback control gain, and the adaptive law of the control gain is adapted online to estimate parametric mismatch. Based on the Lyapunov stability theory, the AFTSFC guarantees that the error of synchronization is uniformly ultimately bounded (UUB); i.e., the drive and response chaotic systems can be synchronized with only a small bounded error. The simulation results demonstrate that the proposed method is able to provide a satisfactory synchronization performance.
\end{abstract}

Keywords: Chaotic System, LMI, Terminal Sliding-Function, T-S Fuzzy Model

\section{Introduction}

It is well known that synchronization of chaotic systems faces some specific problems, such as being extremely sensitive to different initial conditions between the drive and response systems, and having uncertain parameters and parameter mismatch between both systems. Recently, several methods have been proposed in the literature to synchronize chaotic systems, such as those based on fuzzy mode control [1-4], LMI technique [6-8], sliding-mode control [9-11], state observer based design [12, 13], and adaptive control [14, 15]. All of these have had practical applications in secure communication.

The T-S fuzzy-model-based control technique is exact and effective in the control of chaotic systems. In [6], the most chaotic systems can be exactly represented by T-S fuzzy models with simple rules. Moreover, LMI algorithms [5-8, 20, 21] have been widely adopted in solving the problem of stability in T-S fuzzy control systems, while adaptive algorithms and SMC techniques have been applied against parameter variations and external disturbances. Recently, several methods combining the robustness of SMC and the intelligence of fuzzy logic and adaptive algorithms have been developed [17-19] to improve controller performance. An adaptive synchronization of uncertain chaotic systems based on T-S fuzzy models is proposed in [15], and it is specifically derived to estimate the uncertain 
parameters or parameter mismatch between the drive and response systems. Guo et al. [8] presents an integral observer-based chaos synchronization approach which uses the integration of the output signal as the drive signal so that the noise performance can be improved.

Another approach, called terminal sliding-mode control (TSMC), has been developed in [22-27]. Different from the classical sliding mode, the terminal sliding mode has a nonlinear sliding surface. While reaching the terminal sliding mode, the system tracking error can converge to zero in finite time. Unfortunately, chattering still remains a problem in terminal sliding mode control.

In this paper, a new scheme of chaos synchronization is presented for a class of chaotic systems, which combines T-S fuzzy terminal sliding-function (FTSF) control and adaptive scheme. The combined scheme preserves the advantages of both of these methods. The proposed scheme uses T-S fuzzy models to exactly represent the system dynamics of chaotic systems. An appropriate FTSF is designed and then applied to the control law. By using the Lyapunov stability criterion, the LMI stability condition can be derived and the adaptive law of control gain can be defined to realize the synchronization. The initial feedback gain can be obtained by solving the LMI problem so that the initial synchronization performance can be improved. The adaptive scheme does not require prior knowledge of dynamic parameters and can adjust the feedback gain of the controller to reduce the error between the drive and the response of chaotic systems. Based on the Lyapunov approach, the proposed AFTSFC guarantees that the tracking error and the FTSF are UUB.

This paper is organized as follows. In Section 2, the AFTSFC scheme is presented. In Section 3, examples and corresponding numerical results are shown to demonstrate the performance of the proposed approach. Three examples with parameter mismatch chaotic systems are considered in the synchronization. Finally, the conclusions are presented in Section 4.

\section{Chaotic Synchronization Using AFTSFC}

Based on the T-S fuzzy model of chaotic systems, three types of chaotic synchronization are proposed in this paper. The synchronization problem forces the response system to the same internal state as in the drive system. In this section a new AFTSFC scheme is proposed for chaos synchronization so that the parameter mismatch performance can be improved. The block diagram of the synchronization scheme is shown in Figure 1. The details of the T-S fuzzy model, T-S fuzzy terminal sliding function, adaptive scheme, and fuzzy controller are presented in the following subsections.

\subsection{T-S fuzzy model}

In order to realize a fuzzy system design based on LMI algorithms, chaotic systems and terminal sliding surface should be represented via T-S fuzzy models. In prior research [6], several chaotic systems have been represented exactly by the T-S fuzzy model, which is described by fuzzy if-then rules where the consequent parts represent linear models. Consider the dynamics of the drive chaotic systems represented as follows:

Drive System Rule $i$ :

$$
\begin{aligned}
& \text { If } \hat{z}_{1} \text { is } \hat{M}_{i 1}, \ldots, \hat{z}_{p^{\prime}} \text { is } \hat{M}_{i p^{\prime}} \text {, then } \\
& \dot{\hat{\mathbf{x}}}=\hat{A}_{i} \hat{\mathbf{x}} .
\end{aligned}
$$




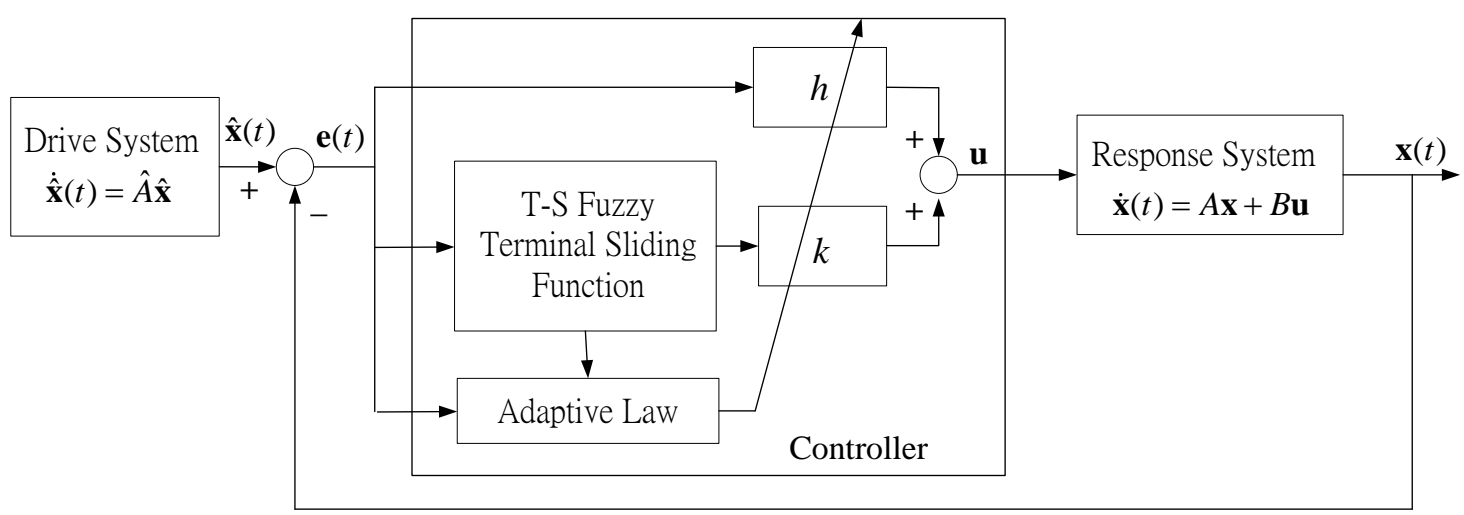

Figure 1. Block diagram of the chaotic synchronization system

$\hat{M}_{i j}\left(j=1,2, \ldots, p^{\prime}\right)$ is a fuzzy term of rule $i$ and $\hat{z}_{j}\left(j=1,2, \ldots, p^{\prime}\right)$ is the premise variable. $\hat{\mathbf{x}}(t) \in R^{n}$ is the state vector, $\hat{A}_{i} \in R^{n \times n}$.

The fuzzy drive system is inferred as follows:

$$
\dot{\hat{\mathbf{x}}}=\sum_{i=1}^{r} \hat{f}_{i}(\hat{z}) \hat{A}_{i} \hat{\mathbf{x}} \quad i=1, \ldots, r
$$

where

$$
\hat{f}_{i}(\hat{z})=\frac{\hat{\xi}_{i}^{f}(\hat{z})}{\sum_{i=1}^{r} \hat{\xi}_{i}^{f}(\hat{z})} .
$$

$\hat{f}_{i}(\hat{z})$ can be regarded as the normalized weight of the if-then rules, and $r$ is the number of fuzzy rules.

$$
\hat{\xi}_{i}^{f}(\hat{z})=\prod_{j=1}^{p^{\prime}} \hat{M}_{i j}\left(\hat{z}_{j}\right)
$$

$\hat{M}_{i j}\left(\hat{z}_{j}\right)$ is the grade of membership of $\hat{z}_{j}$ in $\hat{M}_{i j}$.

Consequently, the dynamics of the response chaotic systems can be represented as follows: Response System Rule $i$ :

If $z_{1}$ is $M_{i 1}, \ldots, z_{p^{\prime}}$ is $M_{i p^{\prime}}$, then

$$
\dot{\mathbf{x}}=A_{i} \mathbf{x}+B_{i} \mathbf{u} .
$$

$M_{i j}\left(j=1,2, \ldots, p^{\prime}\right)$ is a fuzzy term of rule $i$ and $z_{j}\left(j=1,2, \ldots, p^{\prime}\right)$ is the premise variable. $\mathbf{x}(t) \in R^{n}$ is the state vector of the response system, $u(t) \in R^{m}$ is the input vector, $B_{i} \in R^{n \times m}$. $A_{i} \in R^{n \times n}$ are different from those of the drive system, that is, $A_{i} \neq \hat{A}_{i}$.

The fuzzy response system can be inferred as

$$
\dot{\mathbf{x}}=\sum_{i=1}^{r} f_{i}(z)\left(A_{i} \mathbf{x}+B_{i} \mathbf{u}\right)
$$

where 


$$
f_{i}(z)=\frac{\xi_{i}^{f}(z)}{\sum_{i=1}^{r} \xi_{i}^{f}(z)}, \xi_{i}^{f}(z)=\prod_{j=1}^{p^{\prime}} M_{i j}\left(z_{j}\right)
$$

\subsection{T-S Fuzzy Terminal sliding function}

This subsection describes the FTSF that is applied to the control law. The FTSF design can be decoupled into two steps. The first step is the selection of an appropriate TSF. The next step, the TSF and control law will be represented by the T-S fuzzy model, respectively.

Let the synchronization error signal be

$$
\mathbf{e}=\hat{\mathbf{x}}-\mathbf{x} .
$$

where $\mathbf{e}=\left[e_{1}, \ldots, e_{i}, \ldots, e_{n}\right], \hat{\mathbf{x}}=\left[\hat{x}_{1}, \ldots, \hat{x}_{i}, \ldots, \hat{x}_{n}\right]$, and $\mathbf{x}=\left[x_{1}, \ldots, x_{i}, \ldots, x_{n}\right]$.

The control goal considered in this paper is that the drive and response chaotic systems can be synchronized with only a small bounded error.

The TSF is defined as

$$
\mathbf{s}=\alpha \mathbf{e}+\int_{0}^{t} \beta \mathbf{e}^{q / p} d \tau
$$

where $\alpha=\operatorname{diag}\left[\alpha_{1}, \ldots, \alpha_{i}, \ldots, \alpha_{n}\right]$ and $\beta=\operatorname{diag}\left[\beta_{1}, \ldots, \beta_{i}, \ldots, \beta_{n}\right]$ are positive constants, while $p$ and $q$ are positive odd integers and satisfy the following conditions [23],

$$
p>q \quad \text { and } \quad 2 q>p .
$$

The FTSF and FTSF dynamic are constructed as follows.

From (9), the time derivative of $\mathbf{s}$ becomes

$$
\dot{\mathbf{s}}=\alpha \dot{\mathbf{e}}+\beta \mathbf{e}^{q / p} .
$$

The terminal sliding-mode is then defined as $\mathbf{s}=0$ and $\dot{\mathbf{s}}=0$, and can be expressed in the following equations

$$
\begin{aligned}
& \alpha \mathbf{e}+\int_{0}^{t} \beta \mathbf{e}^{q / p} d \tau=0 \\
& \alpha \dot{\mathbf{e}}+\beta \mathbf{e}^{q / p}=0 .
\end{aligned}
$$

The slope of the tangent line at a point $e_{i l}$ on the curve $\alpha_{i} \dot{e}_{i}+\beta_{i} e_{i}^{q / p}=0$ is given as follows:

$$
m_{i l}=-\beta_{i} \Gamma_{i l}
$$

where $\Gamma_{i l}=\frac{q}{\alpha_{i} p} e_{i l}^{(q-p) / p}$.

The equation of the slope at the point $e_{i l}$ is

$$
\alpha_{i} \dot{e}_{i}+\beta_{i} \Gamma_{i l} e_{i}=0
$$


The phase plot of the $\dot{\mathbf{s}}$ is shown in Figure 2. From (14), the T-S fuzzy terminal sliding dynamic $\dot{\mathbf{s}}$ is given as follows.

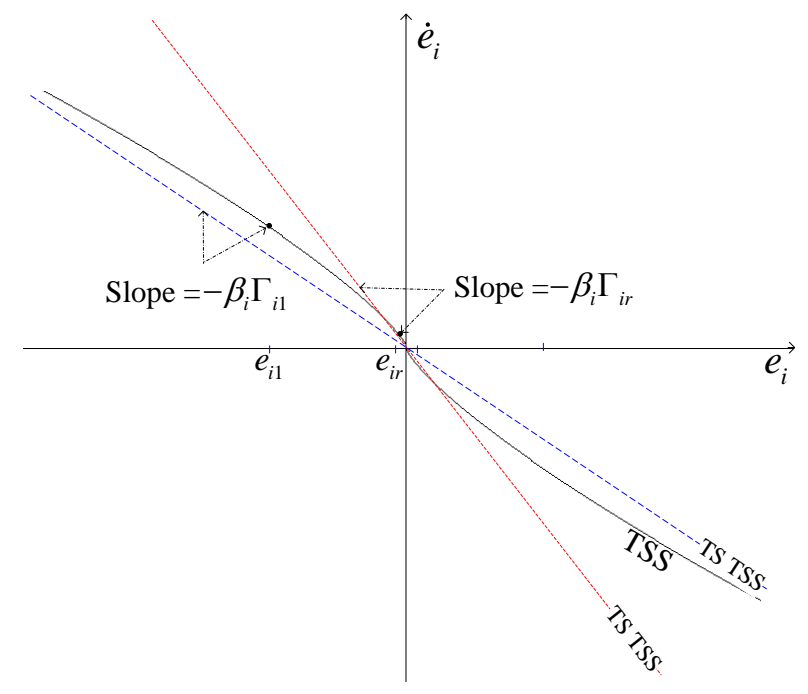

Figure 2. Phase plot of the $\dot{s}_{i}$

Rule $i$ :

If $z_{1}^{\prime \prime}$ is $M_{i 1}^{\prime \prime}, \ldots, z_{p^{\prime}}^{\prime \prime}$ is $M_{i p^{\prime}}^{\prime \prime}$, then

$$
\dot{\mathbf{s}}=\alpha \dot{\mathbf{e}}+\beta \Gamma_{l} \mathbf{e}, \quad l=1, \ldots, r .
$$

where $\Gamma_{l}=\left[\Gamma_{1 l}, \ldots, \Gamma_{i l}, \ldots, \Gamma_{n l}\right]$.

The $\dot{\mathbf{s}}$ can be inferred as

$$
\dot{\mathbf{s}}=\sum_{l=1}^{r} \mu_{l}\left(z^{\prime \prime}\right)\left(\alpha \dot{\mathbf{e}}+\beta \Gamma_{l} \mathbf{e}\right)
$$

where $\mu_{l}\left(z^{\prime \prime}\right)=\frac{\xi_{l}^{\mu}\left(z^{\prime \prime}\right)}{\sum_{l=1}^{r} \xi_{l}^{\mu}\left(z^{\prime \prime}\right)}, \xi_{i}^{\mu}\left(z^{\prime \prime}\right)=\prod_{j=1}^{p^{\prime}} M_{i j}^{\prime \prime}\left(z_{j}^{\prime \prime}\right)$.

T-S fuzzy terminal sliding function can be inferred as

$$
\mathbf{s}=\sum_{l=1}^{r} \mu_{l}\left(z^{\prime \prime}\right)\left(\alpha \mathbf{e}+\beta \Gamma_{l} \int_{0}^{t} \mathbf{e} \cdot d t\right) .
$$

Let us design the control law as follows.

Control Rule $i$ :

$$
\text { If } \begin{array}{r}
z_{1}^{\prime} \text { is } M_{i 1}^{\prime}, \ldots, z_{p^{\prime}}^{\prime} \text { is } M_{i p^{\prime}}^{\prime}, \text { then } \\
\mathbf{u}=k_{i} \mathbf{s}+h_{i} \mathbf{e}, \quad i=1, \ldots, r^{\prime}
\end{array}
$$

where $k_{i}$ and $h_{i}$ are adaptive gains. 
The control law can be inferred as

$$
\mathbf{u}=\sum_{i=1}^{r^{\prime}} g_{i}\left(z^{\prime}\right)\left(k_{i} \mathbf{S}+h_{i} \mathbf{e}\right)=k \mathbf{s}+h \mathbf{e}
$$

where

$$
g_{i}\left(z^{\prime}\right)=\frac{\xi_{i}^{g}\left(z^{\prime}\right)}{\sum_{i=1}^{r^{\prime}} \xi_{i}^{g}\left(z^{\prime}\right)}, \xi_{i}^{g}\left(z^{\prime}\right)=\prod_{j=1}^{p^{\prime}} M_{i j}^{\prime}\left(z_{j}^{\prime}\right), k=\sum_{i=1}^{r^{\prime}} g_{i}\left(z^{\prime}\right) k_{i}, h=\sum_{i=1}^{r^{\prime}} g_{i}\left(z^{\prime}\right) h_{i} .
$$

From (2), (6), (8), and (19), the error dynamic system is constructed as

$$
\begin{aligned}
& \dot{\mathbf{e}}=\dot{\hat{\mathbf{x}}}-\dot{\mathbf{x}} \\
= & \sum_{i=1}^{r} \hat{f}_{i}(\hat{z}) \hat{A_{i}} \hat{\mathbf{x}}-\sum_{i=1}^{r} f_{i}(z)\left(A_{i} \mathbf{x}+B_{i} \mathbf{u}\right) \\
= & \sum_{i=1}^{r} \hat{f}_{i}(\hat{z}) \hat{A}_{i} \hat{\mathbf{x}}-\sum_{i=1}^{r} f_{i}(z) A_{i} \mathbf{x}-\sum_{i=1}^{r} \sum_{j=1}^{r^{\prime}} f_{i}(z) g_{j}\left(z^{\prime}\right)\left(B_{i} k_{j} \mathbf{s}+B_{i} h_{j} \mathbf{e}\right) \\
= & \sum_{i=1}^{r} \hat{f}_{i}(\hat{z}) \hat{A}_{i} \hat{\mathbf{x}}-\sum_{i=1}^{r} f_{i}(z) A_{i} \mathbf{x}-\sum_{i=1}^{r} \sum_{j=1}^{r^{\prime}} f_{i}(z) g_{j}\left(z^{\prime}\right)\left(B_{i} k_{j} \mathbf{s}+B_{i} h_{j} \mathbf{e}\right)+\sum_{i=1}^{r} f_{i}(z)\left(A_{i} \hat{\mathbf{x}}-A_{i} \hat{\mathbf{x}}\right) \\
= & \sum_{i=1}^{r} f_{i}(z) A_{i}(\hat{\mathbf{x}}-\mathbf{x})-\sum_{i=1}^{r} \sum_{j=1}^{r^{\prime}} f_{i}(z) g_{j}\left(z^{\prime}\right)\left(B_{i} k_{j} \mathbf{s}+B_{i} h_{j} \mathbf{e}\right)+\sum_{i=1}^{r} \hat{f}_{i}(\hat{z}) \hat{A}_{i} \hat{\mathbf{x}}-\sum_{i=1}^{r} f_{i}(z) A_{i} \hat{\mathbf{x}} \\
= & \sum_{i=1}^{r} \sum_{j=1}^{r^{\prime}} f_{i}(z) g_{j}\left(z^{\prime}\right)\left[\left(A_{i}-B_{i} h_{j}\right) \mathbf{e}-B_{i} k_{j} \mathbf{s}\right]+\mathbf{\omega}
\end{aligned}
$$

where $\boldsymbol{\omega}=\sum_{i=1}^{r}\left(\hat{f}_{i}(\hat{z}) \hat{A}_{i} \hat{\mathbf{x}}-f_{i}(z) A_{i} \hat{\mathbf{x}}\right)$.

It should be noted that $\boldsymbol{\omega}$ is bounded due to that $f_{i}(z), \hat{f}_{i}(\hat{z})$, and $\hat{\mathbf{x}}$ are bounded. From (16) and (21), the $\dot{\mathbf{s}}$ is constructed as

$$
\begin{aligned}
\dot{\mathbf{s}} & =\sum_{i=1}^{r} \sum_{j=1}^{r^{\prime}} \sum_{l=1}^{r} f_{i}(z) g_{j}\left(z^{\prime}\right) \mu_{l}\left(z^{\prime \prime}\right)\left[\left(\alpha A_{i}-\alpha B_{i} h_{j}\right) \mathbf{e}-\alpha B_{i} k_{j} \mathbf{s}+\beta \Gamma_{l} \mathbf{e}\right]+\alpha \boldsymbol{\omega} \\
& =\sum_{i=1}^{r} \sum_{j=1}^{r^{\prime}} \sum_{l=1}^{r} f_{i j l}(Z)\left[\left(\alpha A_{i}-\alpha B_{i} h_{j}+\beta \Gamma_{l}\right) \mathbf{e}-\alpha B_{i} k_{j} \mathbf{s}\right]+\alpha \mathbf{\omega}
\end{aligned}
$$

where $\sum_{i}^{r} \sum_{j}^{r^{\prime}} \sum_{l}^{r} f_{i j l}(Z)=\sum_{i=1}^{r} \sum_{j=1}^{r^{\prime}} \sum_{l=1}^{r} f_{i}(z) g_{j}\left(z^{\prime}\right) \mu_{l}\left(z^{\prime \prime}\right)$.

\subsection{Stability analysis}

In this subsection, the adaptive law and LMI are designed based on the Lyapunov method, and the stability of the overall system is mathematically proved.

An augmented system can be obtained together with (21) and (22)

$$
\left[\begin{array}{c}
\dot{\mathbf{e}} \\
\dot{\mathbf{s}}
\end{array}\right]=\sum_{i}^{r} \sum_{j}^{r^{\prime}} \sum_{l}^{r} f_{i j l}(Z)\left(\left[\begin{array}{cc}
A_{i}-B_{i} h_{j} & -B_{i} k_{j} \\
\alpha A_{i}-\alpha B_{i} h_{j}+\beta \Gamma_{l} & -\alpha B_{i} k_{j}
\end{array}\right] \cdot\left[\begin{array}{l}
\mathbf{e} \\
\mathbf{s}
\end{array}\right]\right)+\left[\begin{array}{c}
\mathbf{\omega} \\
\alpha \mathbf{\omega}
\end{array}\right]
$$




$$
\begin{aligned}
& =\sum_{i}^{r} \sum_{j}^{r^{\prime}} \sum_{l}^{r} f_{i j l}(Z)\left(\left[\begin{array}{cc}
A_{i} & 0 \\
\alpha A_{i}+\beta \Gamma_{l} & 0
\end{array}\right] \cdot\left[\begin{array}{l}
\mathbf{e} \\
\mathbf{s}
\end{array}\right]-\left[\begin{array}{cc}
B_{i} h_{j} & B_{i} k_{j} \\
\alpha B_{i} h_{j} & \alpha B_{i} k_{j}
\end{array}\right] \cdot\left[\begin{array}{l}
\mathbf{e} \\
\mathbf{s}
\end{array}\right]\right)+\left[\begin{array}{c}
\boldsymbol{\omega} \\
\alpha \boldsymbol{\omega}
\end{array}\right] \\
& =\sum_{i}^{r} \sum_{j}^{r^{\prime}} \sum_{l}^{r} f_{i j l}(Z)\left(\left[\begin{array}{cc}
A_{i} & 0 \\
\alpha A_{i}+\beta \Gamma_{l} & 0
\end{array}\right] \cdot\left[\begin{array}{l}
\mathbf{e} \\
\mathbf{s}
\end{array}\right]-\left[\begin{array}{c}
B_{i} \\
\alpha B_{i}
\end{array}\right]\left[\begin{array}{ll}
h_{j} & k_{j}
\end{array}\right]\left[\begin{array}{l}
\mathbf{e} \\
\mathbf{s}
\end{array}\right]\right)+\left[\begin{array}{c}
\mathbf{\omega} \\
\alpha \boldsymbol{\omega}
\end{array}\right] .
\end{aligned}
$$

Using a new notation

$$
\overline{\mathbf{e}}=\left[\begin{array}{l}
\mathbf{e} \\
\mathbf{s}
\end{array}\right], \bar{A}_{i l}=\left[\begin{array}{cc}
A_{i} & 0 \\
\alpha A_{i}+\beta \Gamma_{l} & 0
\end{array}\right], \bar{B}_{i}=\left[\begin{array}{c}
B_{i} \\
\alpha B_{i}
\end{array}\right], \bar{k}_{j}=\left[\begin{array}{ll}
h_{j} & k_{j}
\end{array}\right], \overline{\mathbf{\omega}}=\left[\begin{array}{c}
\boldsymbol{\omega} \\
\alpha \boldsymbol{\omega}
\end{array}\right] .
$$

where $\bar{k}_{j}=\bar{K}_{j}+\bar{\kappa}_{j}, \bar{K}_{j}$ can be obtained by solving the LMI problem, and $\bar{\kappa}_{j}$ is updated by the adaptive law (25). We thus have

$$
\dot{\overline{\mathbf{e}}}=\sum_{i}^{r} \sum_{j}^{r^{\prime}} \sum_{l}^{r} f_{i j l}(Z)\left(\bar{A}_{i l} \overline{\mathbf{e}}-\bar{B}_{i} \bar{k}_{j} \overline{\mathbf{e}}\right)+\overline{\mathbf{\omega}} \text {. }
$$

Define $\bar{\kappa}_{j}^{*}$ as the optimal estimation gain. Let the error of the optimal estimation gain as

$$
\tilde{\bar{\kappa}}_{j}=\bar{\kappa}_{j}^{*}-\bar{\kappa}_{j} .
$$

Theorem 1: Consider the overall augmented system (24), which consists of the error dynamical system (21), the terminal sliding dynamical system (22), and the fuzzy control law (19). The adjustable gain of the fuzzy controller is updated by

$$
\dot{\bar{\kappa}}_{j}=g_{j}\left(z^{\prime}\right) \sum_{i}^{r} f_{i}(z) \bar{B}_{i}^{T} P \overline{\mathbf{e}} \overline{\mathbf{e}}^{T} .
$$

And a suitable initial feedback gain $\bar{K}_{j}$ is selected such that

$$
\bar{A}_{i l}^{T} P+P \bar{A}_{i l}-\bar{K}_{j}^{T} \bar{B}_{i}^{T} P-P \bar{B}_{i} \bar{K}_{j}-\rho^{2} P P+\gamma^{2} I+2 \gamma \rho P+\rho^{2} P P \leq 0
$$

where $P$ is a positive definite and symmetric matrix, $I$ is the identity matrix, $\gamma>0$, and $\rho>0$. The augmented system state will then converge to the following small neighborhood of the origin:

$$
D_{0}=\left\{\overline{\mathbf{e}}:\|\overline{\mathbf{e}}\| \leq \frac{2 \sigma}{\gamma\left(\gamma+2 \rho \lambda_{\text {min }}(P)\right)}\right\}
$$

where $\lambda_{\min }(P)$ denotes the minimum eigenvalue of matrix $P$, and $\sigma$ is a small positive constant. Then, the AFTSFC guarantees that the synchronization errors are UUB.

\section{Proof:}

To prove the stability of the system, we choose the Lyapunov function candidate as

$$
V=\overline{\mathbf{e}}^{T} P \overline{\mathbf{e}}+\sum_{j}^{r^{\prime}} \operatorname{tr}\left(\tilde{\bar{\kappa}}_{j}^{T} \tilde{\bar{\kappa}}_{j}\right) .
$$

The time derivative of $V$ along the state trajectory becomes 


$$
\begin{aligned}
& \dot{V}=\dot{\overline{\mathbf{e}}}^{T} P \overline{\mathbf{e}}+\overline{\mathbf{e}}^{T} P \dot{\overline{\mathbf{e}}}+\sum_{j}^{r^{\prime}} \operatorname{tr}\left(\dot{\overline{\bar{\kappa}}}_{j}^{T} \tilde{\bar{\kappa}}_{j}+\tilde{\bar{\kappa}}_{j}^{T} \dot{\bar{\kappa}}_{j}\right) \\
& =\sum_{i}^{r} \sum_{j}^{r^{\prime}} \sum_{l}^{r} f_{i j l}(Z)\left(\overline{\mathbf{e}}^{T} \bar{A}_{i l}^{T} P \overline{\mathbf{e}}-\overline{\mathbf{e}}^{T} \bar{k}_{j}^{T} \bar{B}_{i}^{T} P \overline{\mathbf{e}}\right)+\overline{\boldsymbol{\omega}}^{T} P \overline{\mathbf{e}} \\
& +\sum_{i}^{r} \sum_{j}^{r^{\prime}} \sum_{l}^{r} f_{i j l}(Z)\left(\overline{\mathbf{e}}^{T} P \bar{A}_{i l} \overline{\mathbf{e}}-\overline{\mathbf{e}}^{T} P \bar{B}_{i} \bar{k}_{j} \overline{\mathbf{e}}\right)+\overline{\mathbf{e}}^{T} P \overline{\mathbf{\omega}}+\sum_{j}^{r^{\prime}} \operatorname{tr}\left(\dot{\tilde{\bar{\kappa}}}_{j}^{T} \tilde{\bar{\kappa}}_{j}+\tilde{\bar{\kappa}}_{j}^{T} \dot{\bar{\kappa}}_{j}\right) \\
& =\sum_{i}^{r} \sum_{j}^{r^{\prime}} \sum_{l}^{r} f_{i j l}(Z)\left[\overline{\mathbf{e}}^{T}\left(\bar{A}_{i l}^{T} P+P \bar{A}_{i l}-\bar{K}_{j}^{T} \bar{B}_{i}^{T} P-P \bar{B}_{i} \bar{K}_{j}\right) \overline{\mathbf{e}}\right] \\
& +\overline{\mathbf{e}}^{T} P \overline{\mathbf{\omega}}-\sum_{i}^{r} \sum_{j}^{r^{\prime}} f_{i}(z) g_{j}\left(z^{\prime}\right)\left(\overline{\mathbf{e}}^{T} \bar{\kappa}_{j}^{T} \bar{B}_{i}^{T} P \overline{\mathbf{e}}\right) \\
& +\overline{\boldsymbol{\omega}}^{T} P \overline{\mathbf{e}}-\sum_{i}^{r} \sum_{j}^{r^{\prime}} f_{i}(z) g_{j}\left(z^{\prime}\right)\left(\overline{\mathbf{e}}^{T} P \bar{B}_{i} \bar{\kappa}_{j} \overline{\mathbf{e}}\right)+\sum_{j}^{r^{\prime}} \operatorname{tr}\left(\dot{\bar{\kappa}}_{j}^{T} \tilde{\bar{\kappa}}_{j}+\tilde{\bar{\kappa}}_{j}^{T} \dot{\tilde{\bar{\kappa}}}_{j}\right) .
\end{aligned}
$$

Using $\tilde{\bar{\kappa}}_{j}=\bar{\kappa}_{j}^{*}-\bar{\kappa}_{j}$, then the above equation becomes

$$
\begin{aligned}
\dot{V}= & \sum_{i}^{r} \sum_{j}^{r^{\prime}} \sum_{l}^{r} f_{i j l}(Z)\left[\overline{\mathbf{e}}^{T}\left(\bar{A}_{i l}^{T} P+P \bar{A}_{i l}-\bar{K}_{j}^{T} \bar{B}_{i}^{T} P-P \bar{B}_{i} \bar{K}_{j}\right) \overline{\mathbf{e}}\right] \\
& +\overline{\mathbf{e}}^{T} P \overline{\mathbf{\omega}}+\sum_{i}^{r} \sum_{j}^{r^{\prime}} f_{i}(z) g_{j}\left(z^{\prime}\right)\left(\overline{\mathbf{e}}^{T} \tilde{\bar{\kappa}}_{j}^{T} \bar{B}_{i}^{T} P \overline{\mathbf{e}}-\overline{\mathbf{e}}^{T} \overline{\boldsymbol{\kappa}}_{j}^{T^{*}} \bar{B}_{i}^{T} P \overline{\mathbf{e}}\right) \\
& +\overline{\mathbf{\omega}}^{T} P \overline{\mathbf{e}}+\sum_{i}^{r} \sum_{j}^{r^{\prime}} f_{i}(z) g_{j}\left(z^{\prime}\right)\left(\overline{\mathbf{e}}^{T} P \bar{B}_{i} \tilde{\bar{\kappa}}_{j} \overline{\mathbf{e}}^{T} \overline{\mathbf{e}}^{T} P \bar{B}_{i} \overline{\boldsymbol{\kappa}}_{j}^{*} \overline{\mathbf{e}}\right)+\sum_{j}^{r^{\prime}} \operatorname{tr}\left(\dot{\bar{\kappa}}_{j}^{T} \tilde{\bar{\kappa}}_{j}+\tilde{\bar{\kappa}}_{j}^{T} \dot{\bar{\kappa}}_{j}\right) \\
= & \sum_{i}^{r} \sum_{j}^{r^{\prime}} \sum_{l}^{r} f_{i j l}(Z)\left[\overline{\mathbf{e}}^{T}\left(\bar{A}_{i l}^{T} P+P \bar{A}_{i l}-\bar{K}_{j}^{T} \bar{B}_{i}^{T} P-P \bar{B}_{i} \bar{K}_{j}\right) \overline{\mathbf{e}}\right] \\
& +\overline{\mathbf{e}}^{T} P \overline{\mathbf{\omega}}-\sum_{i}^{r} \sum_{j}^{r^{\prime}} f_{i}(z) g_{j}\left(z^{\prime}\right)\left(\overline{\mathbf{e}}^{T} \overline{\boldsymbol{\kappa}}_{j}^{T^{*}} \bar{B}_{i}^{T} P \overline{\mathbf{e}}\right) \\
& +\overline{\mathbf{\omega}}^{T} P \overline{\mathbf{e}}-\sum_{i}^{r} \sum_{j}^{r^{\prime}} f_{i}(z) g_{j}\left(z^{\prime}\right)\left(\overline{\mathbf{e}}^{T} P \bar{B}_{i} \bar{\kappa}_{j}^{*} \overline{\mathbf{e}}\right) \\
& +\sum_{i}^{r} \sum_{j}^{r^{\prime}} f_{i}(z) g_{j}\left(z^{\prime}\right)\left(\overline{\mathbf{e}}^{T} \tilde{\bar{\kappa}}_{j}^{T} \bar{B}_{i}^{T} P \overline{\mathbf{e}}+\overline{\mathbf{e}}^{T} P \bar{B}_{i} \tilde{\bar{\kappa}}_{j} \overline{\mathbf{e}}\right)+\sum_{j}^{r^{\prime}} \operatorname{tr}\left(\dot{\dot{\bar{\kappa}}_{j}^{T}} \tilde{\bar{\kappa}}_{j}+\tilde{\bar{\kappa}}_{j}^{T} \dot{\tilde{\bar{\kappa}}}_{j}\right)
\end{aligned}
$$

Since $\xi=\overline{\boldsymbol{\omega}}^{T} P \overline{\mathbf{e}}-\sum_{i}^{r} \sum_{j}^{r^{\prime}} f_{i}(z) g_{j}\left(z^{\prime}\right)\left(\overline{\mathbf{e}}^{T} P \bar{B}_{i} \overline{\boldsymbol{\kappa}}_{j}^{*} \overline{\mathbf{e}}\right)$

$$
\leq\left\|\overline{\boldsymbol{\omega}}^{T} P\right\|\|\overline{\mathbf{e}}\|-\eta^{*}\|\overline{\mathbf{e}}\|^{2}=\left(\left\|\overline{\boldsymbol{\omega}}^{T} P\right\|-\eta^{*}\|\overline{\mathbf{e}}\|\right)\|\overline{\mathbf{e}}\| \leq \sigma\|\overline{\mathbf{e}}\|
$$

where $\left\|\overline{\boldsymbol{\omega}}^{T} P\right\|$ is bounded, $\sum_{i}^{r} \sum_{j}^{r^{\prime}} f_{i}(z) g_{j}\left(z^{\prime}\right)\left(\overline{\mathbf{e}}^{T} P \bar{B}_{i} \bar{\kappa}_{j}^{*} \overline{\mathbf{e}}\right) \geq \eta^{*}\|\overline{\mathbf{e}}\|^{2}, \eta^{*}>0$, and $\sigma$ is a positive constant. Then (30) becomes

$$
\begin{aligned}
\dot{V} \leq & \sum_{i}^{r} \sum_{j}^{r^{\prime}} \sum_{l}^{r} f_{i j l}(Z)\left[\overline{\mathbf{e}}^{T}\left(\bar{A}_{i l}^{T} P+P \bar{A}_{i l}-\bar{K}_{j}^{T} \bar{B}_{i}^{T} P-P \bar{B}_{i} \bar{K}_{j}\right) \overline{\mathbf{e}}\right] \\
& +2 \sum_{i}^{r} \sum_{j}^{r^{\prime}} f_{i}(z) g_{j}\left(z^{\prime}\right) \overline{\mathbf{e}}^{T} \tilde{\bar{\kappa}}_{j}^{T} \bar{B}_{i}^{T} P \overline{\mathbf{e}}+2 \sum_{j}^{r^{\prime}} \operatorname{tr}\left(\tilde{\bar{\kappa}}_{j}^{T} \dot{\tilde{\bar{\kappa}}}_{j}\right)+2 \sigma\|\overline{\mathbf{e}}\|
\end{aligned}
$$




$$
\begin{aligned}
= & \sum_{i}^{r} \sum_{j}^{r^{\prime}} \sum_{l}^{r} f_{i j l}(Z)\left[\overline{\mathbf{e}}^{T}\left(\bar{A}_{i l}^{T} P+P \bar{A}_{i l}-\bar{K}_{j}^{T} \bar{B}_{i}^{T} P-P \bar{B}_{i} \bar{K}_{j}\right) \overline{\mathbf{e}}\right] \\
& +2 \operatorname{tr}\left(\sum_{j}^{r^{\prime}} \tilde{\bar{\kappa}}_{j}^{T} \dot{\overline{\bar{\kappa}}}_{j}+\sum_{i}^{r} \sum_{j}^{r^{\prime}} f_{i}(z) g_{j}\left(z^{\prime}\right) \tilde{\bar{\kappa}}_{j}^{T} \bar{B}_{i}^{T} P \overline{\mathbf{e}} \overline{\mathbf{e}}^{T}\right)+2 \sigma\|\overline{\mathbf{e}}\| .
\end{aligned}
$$

Using the adaptive law (25), then the above equation becomes

$$
\dot{V} \leq \sum_{i}^{r} \sum_{j}^{r^{\prime}} \sum_{l}^{r} f_{i j l}(Z)\left[\overline{\mathbf{e}}^{T}\left(\bar{A}_{i l}^{T} P+P \bar{A}_{i l}-\bar{K}_{j}^{T} \bar{B}_{i}^{T} P-P \bar{B}_{i} \bar{K}_{j}\right) \overline{\mathbf{e}}\right]+2 \sigma\|\overline{\mathbf{e}}\| .
$$

For given $\gamma>0$, and $\rho>0$, then (32) becomes

$$
\begin{aligned}
\dot{V} \leq & \sum_{i}^{r} \sum_{j}^{r^{\prime}} \sum_{l}^{r} f_{i j l}(Z)\left[\overline { \mathbf { e } } ^ { T } \left(\bar{A}_{i l}^{T} P+P \bar{A}_{i l}-\bar{K}_{j}^{T} \bar{B}_{i}^{T} P-P \bar{B}_{i} \bar{K}_{j}\right.\right. \\
& \left.\left.-\rho^{2} P P+\gamma^{2} I+2 \gamma \rho P+\rho^{2} P P\right) \overline{\mathbf{e}}\right]-\gamma^{2} \overline{\mathbf{e}}^{T} \overline{\mathbf{e}}-2 \gamma \rho \overline{\mathbf{e}}^{T} P \overline{\mathbf{e}}+2 \sigma\|\overline{\mathbf{e}}\| .
\end{aligned}
$$

If (34) is satisfied

$$
\bar{A}_{i l}^{T} P+P \bar{A}_{i l}-\bar{K}_{j}^{T} \bar{B}_{i}^{T} P-P \bar{B}_{i} \bar{K}_{j}-\rho^{2} P P+\gamma^{2} I+2 \gamma \rho P+\rho^{2} P P \leq 0
$$

then we get

$$
\begin{aligned}
\dot{V} & \leq-\gamma^{2} \overline{\mathbf{e}}^{T} \overline{\mathbf{e}}-2 \gamma \rho \overline{\mathbf{e}}^{T} P \overline{\mathbf{e}}+2 \sigma\|\overline{\mathbf{e}}\| \\
& \leq-\gamma^{2}\|\overline{\mathbf{e}}\|^{2}-2 \gamma \rho \lambda_{\text {min }}(P)\|\overline{\mathbf{e}}\|^{2}+2 \sigma\|\overline{\mathbf{e}}\| \\
& =-\gamma\|\overline{\mathbf{e}}\|\left(\gamma\|\overline{\mathbf{e}}\|+2 \rho \lambda_{\text {min }}(P)\|\overline{\mathbf{e}}\|-\frac{2 \sigma}{\gamma}\right) \\
& =-\gamma\|\overline{\mathbf{e}}\|\left[\left(\gamma+2 \rho \lambda_{\text {min }}(P)\right)\|\overline{\mathbf{e}}\|-\frac{2 \sigma}{\gamma}\right] .
\end{aligned}
$$

If the error variable $\overline{\mathbf{e}}$ does not belong to $D_{0}$, namely, $\|\overline{\mathbf{e}}\| \geq 2 \sigma / \gamma\left(\gamma+2 \rho \lambda_{\min }(P)\right)$, then we have

$$
\dot{V} \leq-\gamma\|\overline{\mathbf{e}}\| \quad \forall \quad\|\overline{\mathbf{e}}\| \geq \frac{2 \sigma}{\gamma\left(\gamma+2 \rho \lambda_{\text {min }}(P)\right)} .
$$

Based on the Lyapunov stability theory, the AFTSFC guarantees that the states of the augmented system (23) are UUB; i.e., the augmented system will converge to a small neighborhood of the origin. By choosing $\gamma$ and $\rho$ appropriately, the state trajectories can be synchronized with only a small bounded error. The proof is thus completed.

The projection operators (37) are used to modify the adaptive law (25) such that the parameters vector will remain inside the constraint set $\Xi_{\bar{\kappa}_{j}}=\left\{\bar{\kappa}_{j} \mid\left\|\bar{\kappa}_{j}\right\| \leq M_{\bar{\kappa}_{j}}\right\}$.

$$
\dot{\bar{\kappa}}_{j}=P\left[g_{j}(z) \sum_{i}^{r} f_{i}(z) \bar{B}_{i}^{T} P \overline{\mathbf{e}} \overline{\mathbf{e}}^{T}\right]
$$




$$
=g_{j}(z)\left[\sum_{i}^{r} f_{i}(z) \bar{B}_{i}^{T} P \overline{\mathbf{e}} \overline{\mathbf{e}}^{T}-I_{\bar{\kappa}_{j}} \frac{\operatorname{tr}\left(\sum_{i}^{r} f_{i}(z) \bar{\kappa}_{j}^{T} \bar{B}_{i}^{T} P \overline{\mathbf{e}} \overline{\mathbf{e}}^{T}\right)}{\operatorname{tr}\left(\bar{\kappa}_{j}^{T} \bar{\kappa}_{j}\right)} \bar{\kappa}_{j}\right]
$$

where

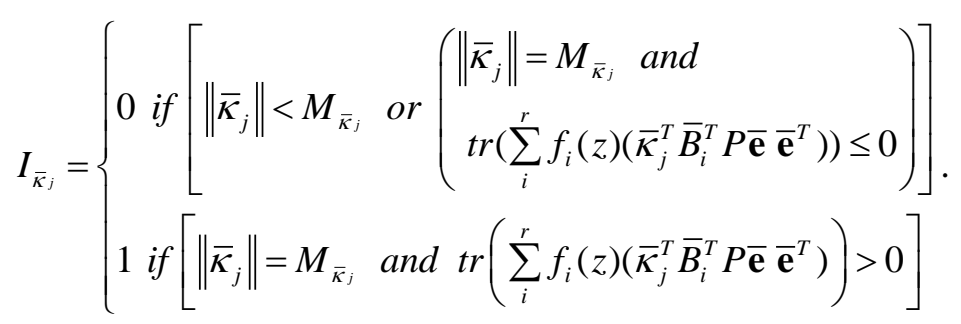

There exists a symmetric positive-definite matrix $P$ such that by pre-multiplying and post-multiplying (34) by $P^{-1}$, and defining $Q=P^{-1}$, we can obtain

$$
Q \bar{A}_{i l}^{T}+\bar{A}_{i l} Q-Q \bar{K}_{j}^{T} \bar{B}_{i}^{T}-\bar{B}_{i} \bar{K}_{j} Q-\rho^{2} I+\gamma^{2} Q Q+2 \gamma \rho Q+\rho^{2} I \leq 0 .
$$

Let $\bar{M}_{j}=\bar{K}_{j} Q$, and we can then obtain

$$
Q \bar{A}_{i l}^{T}+\bar{A}_{i l} Q-\bar{M}_{j}^{T} \bar{B}_{i}^{T}-\bar{B}_{i} \bar{M}_{j}-\rho^{2} I+\gamma^{2}\left(Q+\frac{\rho}{\gamma} I\right) I\left(Q+\frac{\rho}{\gamma} I\right) \leq 0 .
$$

Using Schur Complements [16] (39) can be transformed to the following LMI form.

$$
\left[\begin{array}{cc}
Q \bar{A}_{i l}^{T}+\bar{A}_{i l} Q-\bar{M}_{j}^{T} \bar{B}_{i}^{T}-\bar{B}_{i} \bar{M}_{j}-\rho^{2} I & Q+\frac{\rho}{\gamma} I \\
Q+\frac{\rho}{\gamma} I & -\gamma^{2} I
\end{array}\right] \leq 0 .
$$

If suitable matrices $Q$ and $\bar{M}_{j}$ are selected such that the LMI (40) is satisfied, then the feedback gain $\bar{K}_{j}=\bar{M}_{j} Q^{-1}$.

\section{Numerical Examples}

Three examples are given in this section to illustrate the effectiveness of the proposed approach. In example 1, two Rössler systems subject to parameter mismatch are used as the response and drive chaotic systems, while the proposed controller is employed to realize the synchronization. In example 2, two unified chaotic systems are considered as the drive and response systems. In example 3, two different chaotic systems are synchronized using the proposed method, with a unified chaotic system and a Rössler system considered as the response and drive systems, respectively. In all examples, we apply the proposed AFTSFC and adaptive T-S fuzzy classical sliding-function controller (AFSFC) to the chaotic systems, respectively. 


\subsection{Example 1}

The Rössler chaotic drive system is described as follows [1].

$$
\dot{\hat{\mathbf{x}}}=\hat{A} \hat{\mathbf{x}}
$$

where $\hat{\mathbf{x}}=\left[\begin{array}{lll}\hat{x}_{1} & \hat{x}_{2} & \hat{x}_{3}\end{array}\right]^{T}$ and

$$
\hat{A}=\left[\begin{array}{ccc}
0 & -1 & -1 \\
1 & \hat{a} & 0 \\
\hat{b} & 0 & -\left(\hat{c}(t)-\hat{x}_{1}(t)\right)
\end{array}\right], \hat{c}(t)=\frac{\hat{c}_{\max }+\hat{c}_{\min }}{2}+\frac{\hat{c}_{\max }-\hat{c}_{\min }}{2} \sin (t) \in\left[\begin{array}{ll}
\hat{c}_{\min } & \hat{c}_{\max }
\end{array}\right]>0 .
$$

By defining two fuzzy sets, we can obtain the following fuzzy response system that exactly represents the nonlinear equation. The fuzzy Rössler chaotic response system is described as follows.

If $x_{1}$ is $M_{i}$, then

$$
\dot{\mathbf{x}}=A_{i} \mathbf{x}+B_{i} \mathbf{u}
$$

where $\mathbf{x}=\left[\begin{array}{lll}x_{1} & x_{2} & x_{3}\end{array}\right]^{T}, \mathbf{u}=\left[\begin{array}{ll}u_{1} & u_{2}\end{array}\right]^{T}$,

$$
A_{1}=\left[\begin{array}{ccc}
0 & -1 & -1 \\
1 & a & 0 \\
b & 0 & -d
\end{array}\right], A_{2}=\left[\begin{array}{ccc}
0 & -1 & -1 \\
1 & a & 0 \\
b & 0 & d
\end{array}\right] \text {, and } B_{1}=B_{2}=\left[\begin{array}{ll}
0 & 0 \\
1 & 0 \\
0 & 1
\end{array}\right] \text {. }
$$

We assume that $x_{1} \in\left[\begin{array}{ll}-d & d\end{array}\right]$ with $d=25$, and the membership functions are designed as $M_{1}\left(x_{1}\right)=\frac{1}{2}\left(1+\frac{c(t)-x_{1}}{d}\right), M_{2}\left(x_{1}\right)=1-M_{1}\left(x_{1}\right)$.

The TSF is defined as

$$
\mathbf{S}=\alpha \mathbf{e}+\int_{0}^{t} \beta \mathbf{e}^{q / p} d \tau
$$

where $\alpha=\operatorname{diag}\left[\begin{array}{lll}1 & 1 & 1\end{array}\right], \beta=\operatorname{diag}\left[\begin{array}{lll}70 & 70 & 70\end{array}\right], p=9$, and $q=5$. By defining two fuzzy sets, we can represent the FTSF. The fuzzy $\mathbf{s}$ is given as follows.

If $\|\mathbf{e}\|$ is $\left\|\mathbf{e}_{0 l}\right\|$, then

$$
\mathbf{S}=\alpha \mathbf{e}+\beta \Gamma_{l} \int_{0}^{t} \mathbf{e} \cdot d t
$$

where

$$
\begin{aligned}
& \mathbf{e}_{01}=\left[\begin{array}{lll}
\frac{1}{\sqrt{3}} & \frac{1}{\sqrt{3}} & \frac{1}{\sqrt{3}}
\end{array}\right]^{T}, \mathbf{e}_{02}=\left[\begin{array}{lll}
\frac{0.05}{\sqrt{3}} & \frac{0.05}{\sqrt{3}} & \frac{0.05}{\sqrt{3}}
\end{array}\right]^{T},\left\|\mathbf{e}_{01}\right\|=1,\left\|\mathbf{e}_{02}\right\|=0.05, \\
& \Gamma_{1}=\operatorname{diag}\left[\begin{array}{llll}
0.7 & 0.7 & 0.7
\end{array}\right], \text { and } \Gamma_{2}=\operatorname{diag}\left[\begin{array}{lll}
3.5 & 3.5 & 3.5
\end{array}\right]
\end{aligned}
$$

The fuzzy $\dot{\mathbf{s}}$ is given as follows. 


$$
\begin{array}{r}
\text { If }\|\mathbf{e}\| \text { is }\left\|\mathbf{e}_{0 l}\right\| \text {, then } \\
\dot{\mathbf{s}}=\alpha \dot{\mathbf{e}}+\beta \Gamma_{l} \mathbf{e}
\end{array}
$$

The membership functions are shown in Figure 3. The fuzzy state-feedback controller is given as follows.

If $x_{1}$ is $M_{i}$ and $\|\mathbf{e}\|$ is $\left\|\mathbf{e}_{0 l}\right\|$, then

$$
\mathbf{u}=k_{j} \mathbf{s}+h_{j} \mathbf{e}, \quad j=1, \ldots, 4 .
$$

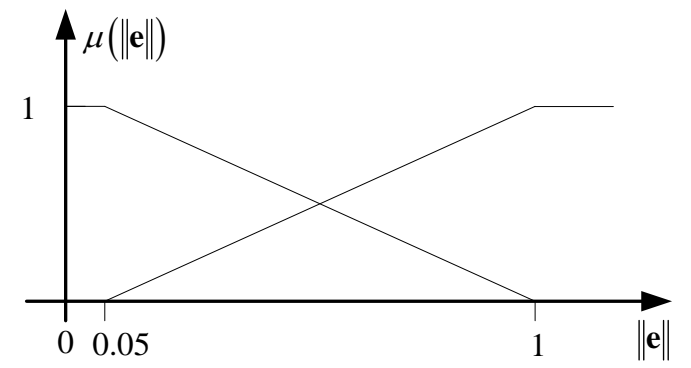

Figure 3. The membership function of $\|\mathbf{e}\|$

In the adaptive T-S fuzzy classical sliding-function controller, the sliding function is defined

$$
\mathbf{S}=\alpha \mathbf{e}+\int_{0}^{t} \beta \Gamma_{1} \cdot \mathbf{e} d \tau
$$

The initial states of the drive and response systems are $\hat{\mathbf{x}}=\left[\begin{array}{lll}0 & 1 & 0\end{array}\right]^{T}$ and $\mathbf{x}=\left[\begin{array}{lll}0 & 0 & 0\end{array}\right]^{T}$, respectively. In the response system, we choose $c=\frac{c_{2}+c_{1}}{2}+\frac{\left(c_{2}-c_{1}\right)}{2} \cos (t)$, $c_{1}=4.5, c_{2}=7.7, a=0.34, b=0.4$, and in the drive system we choose $\hat{a}=a+\frac{a}{2} \sin (t)$, $\hat{b}=b+\frac{b}{2} \sin (t), \hat{c}=\frac{\hat{c}_{2}+\hat{c}_{1}}{2}+\frac{\left(\hat{c}_{2}-\hat{c}_{1}\right)}{2} \sin (t), \hat{c}_{1}=c_{1}+\frac{c_{1}}{2} \sin (t), \hat{c}_{2}=c_{2}+\frac{c_{2}}{2} \sin (t)$, and this is regarded as the parameter mismatch and parameter uncertainty. By choosing $\gamma=10$ and $\rho=1, \bar{M}_{j}$ and $Q$ can be obtained from (40) using the MATLAB LMI toolbox. Then, the feedback gain can be obtained from $\bar{K}_{j}=\bar{M}_{j} Q^{-1}$. The matrix $Q$ is listed as follow: 


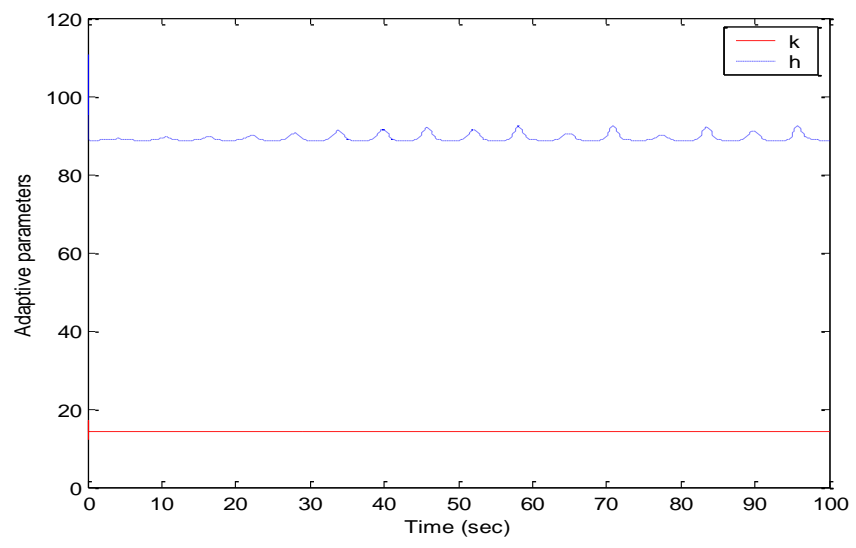

Figure 4. Curves of adaptive gains $\|h\|$ and $\|k\|$
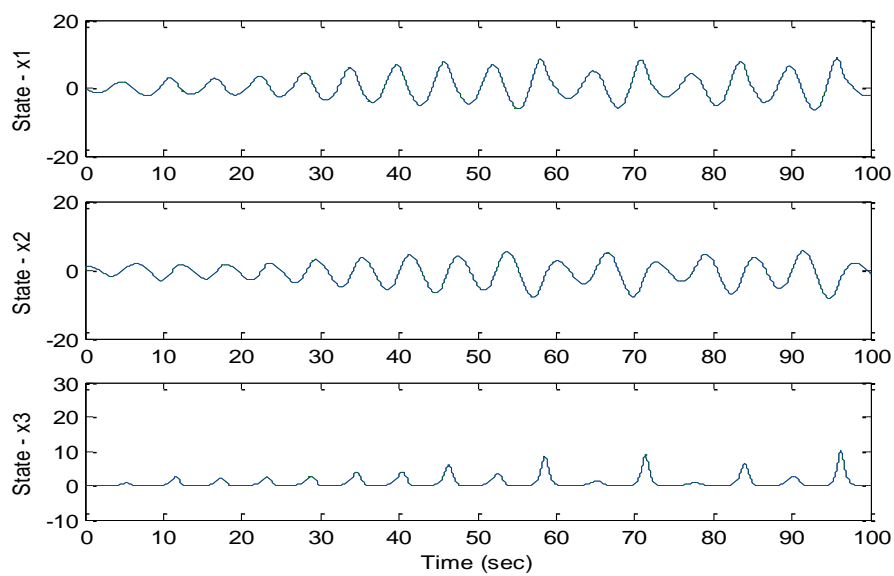

Figure 5(a). The trajectories of the drive (solid line) and response (dashdot line) system under the proposed AFTSFC
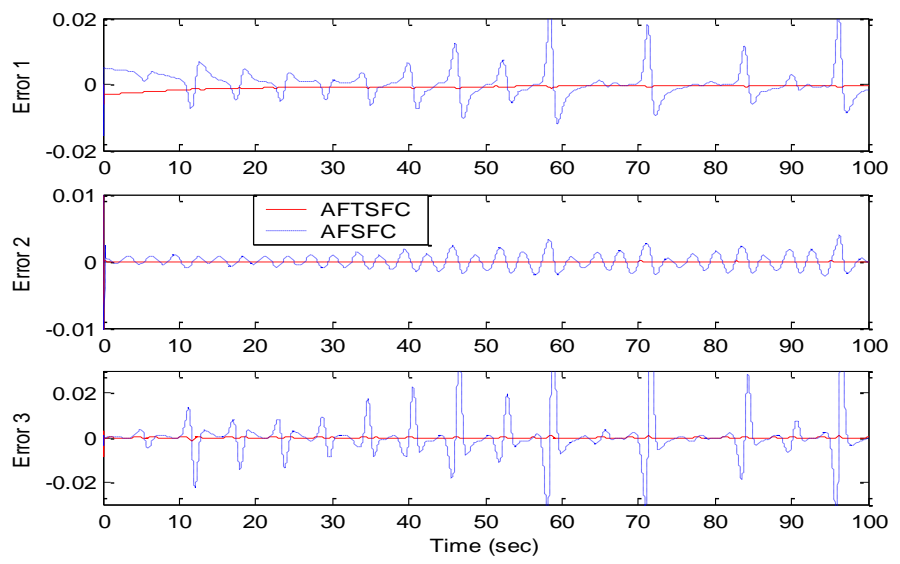

Figure 5(b). The tracking errors of the drive and response systems under the AFTSFC (solid line) and the AFSFC (dashdot line) 


$$
Q=\left[\begin{array}{cccccc}
0.0350 & 0.0020 & 0.0013 & -0.2854 & -0.0203 & -0.0154 \\
0.0020 & 0.1277 & -0.0005 & 0.0112 & -0.6062 & 0.0011 \\
0.0013 & -0.0005 & 0.0738 & 0.0035 & 0.0017 & -0.3673 \\
-0.2854 & 0.0112 & 0.0035 & 4.3546 & 0.0635 & 0.0613 \\
-0.0203 & -0.6062 & 0.0017 & 0.0635 & 3.7970 & 0.0016 \\
-0.0154 & 0.0011 & -0.3673 & 0.0613 & 0.0016 & 2.9721
\end{array}\right] .
$$

Using the adaptive law (25) and (23), we can obtain $k$ and $h$ at $t=100 \mathrm{sec}$ as follows:

$$
k=\left[\begin{array}{ccc}
-0.4351 & 14.2464 & 0.0723 \\
-0.2308 & 0.0784 & 10.6531
\end{array}\right], h=\left[\begin{array}{ccc}
3.7535 & 88.4688 & 0.7610 \\
2.9223 & 0.7035 & 84.8910
\end{array}\right] .
$$

The curves of the adaptive gains $\|h\|$ and $\|k\|$ are shown in Figure 4. The trajectories of the drive and response systems under the proposed AFTSFC are shown in Figure 5a. The tracking errors of the drive and response systems under these two controllers are shown in Figure 5b.

\subsection{Example 2}

The unified chaotic drive system is described as follows [6].

$$
\dot{\hat{\mathbf{x}}}=\hat{A} \hat{\mathbf{x}}
$$

where $\hat{\mathbf{x}}=\left[\begin{array}{lll}\hat{x}_{1} & \hat{x}_{2} & \hat{x}_{3}\end{array}\right]^{T}, \quad \hat{A}=\left[\begin{array}{ccc}-(25 \hat{a}+10) & 25 \hat{a}+10 & 0 \\ 28-35 \hat{a} & 29 \hat{a}-1 & -x_{1} \\ 0 & x_{1} & -\frac{8+\hat{a}}{3}\end{array}\right]$, with $\hat{a} \in\left[\begin{array}{ll}0 & 1\end{array}\right]$.

By defining two fuzzy sets, we can obtain the following fuzzy response system that exactly represents the nonlinear equation. The fuzzy unified chaotic response system is described as follows.

If $x_{1}$ is $M_{i}$, then

$$
\dot{\mathbf{x}}=A_{i} \mathbf{x}+B_{i} \mathbf{u}
$$

where $\mathbf{x}=\left[\begin{array}{lll}x_{1} & x_{2} & x_{3}\end{array}\right]^{T}, \mathbf{u}=\left[\begin{array}{lll}u_{1} & u_{2} & u_{3}\end{array}\right]^{T}, B_{1}=B_{2}=\operatorname{diag}\left[\begin{array}{lll}1 & 1 & 1\end{array}\right]$,

$$
A_{1}=\left[\begin{array}{ccc}
-(25 a+10) & 25 a+10 & 0 \\
28-35 a & 29 a-1 & -d \\
0 & d & -\frac{8+a}{3}
\end{array}\right], \quad A_{2}=\left[\begin{array}{ccc}
-(25 a+10) & 25 a+10 & 0 \\
28-35 a & 29 a-1 & d \\
0 & -d & -\frac{8+a}{3}
\end{array}\right] .
$$

The membership functions are designed as

$$
M_{1}\left(x_{1}\right)=\frac{1}{2}\left(1+\frac{x_{1}}{d}\right), M_{2}\left(x_{1}\right)=\frac{1}{2}\left(1-\frac{x_{1}}{d}\right) .
$$


We assume $x_{1} \in\left[\begin{array}{ll}-d & d\end{array}\right]$, with $d=30$.

In this example, the TSF is defined as (43), and the FTSF is given as (44). The membership functions are shown in Figure 3. The fuzzy state-feedback controller is given as (46). In the AFSFC, the classical sliding function is defined as (47). The initial states of the drive and response systems are $\hat{\mathbf{x}}=\left[\begin{array}{lll}-2 & -2 & 1\end{array}\right]^{T}$ and $\mathbf{x}=\left[\begin{array}{lll}2 & 2 & 6\end{array}\right]^{T}$, respectively. The parameters of the response system are held at $a=0.5$, the parameters of the drive system are changed as $\hat{a}=a+\frac{a}{2} \sin (t)$, and this is regarded as a parameter mismatch. By choosing $\gamma=10$ and $\rho=1$, $\bar{M}_{j}$ and $Q$ can be obtained from (40) using the MATLAB LMI toolbox. Then, the feedback
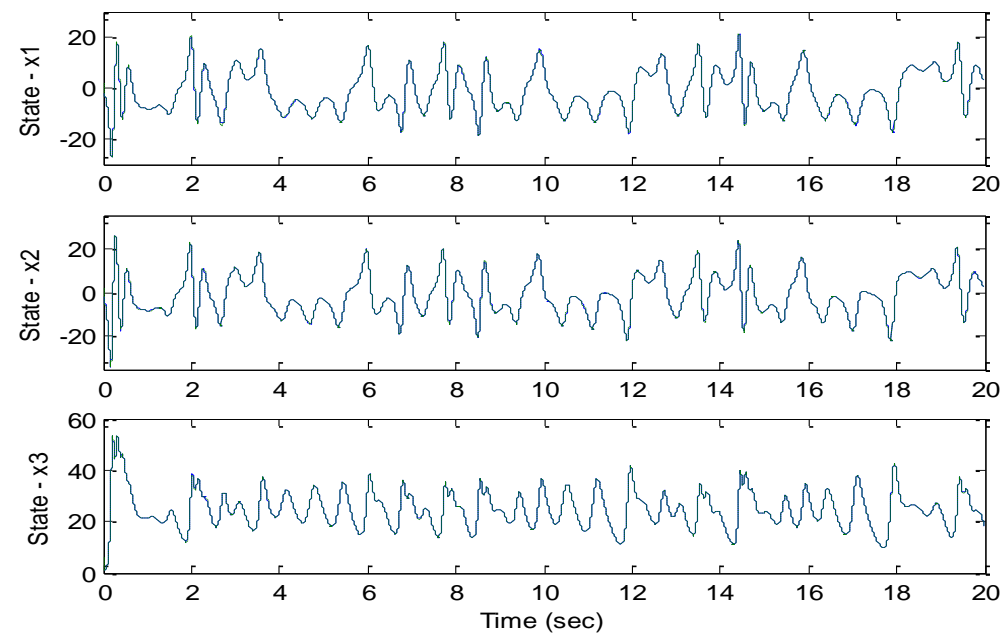

Figure 6(a). The trajectories of the drive (solid line) and response (dashdot line) system under the proposed AFTSFC
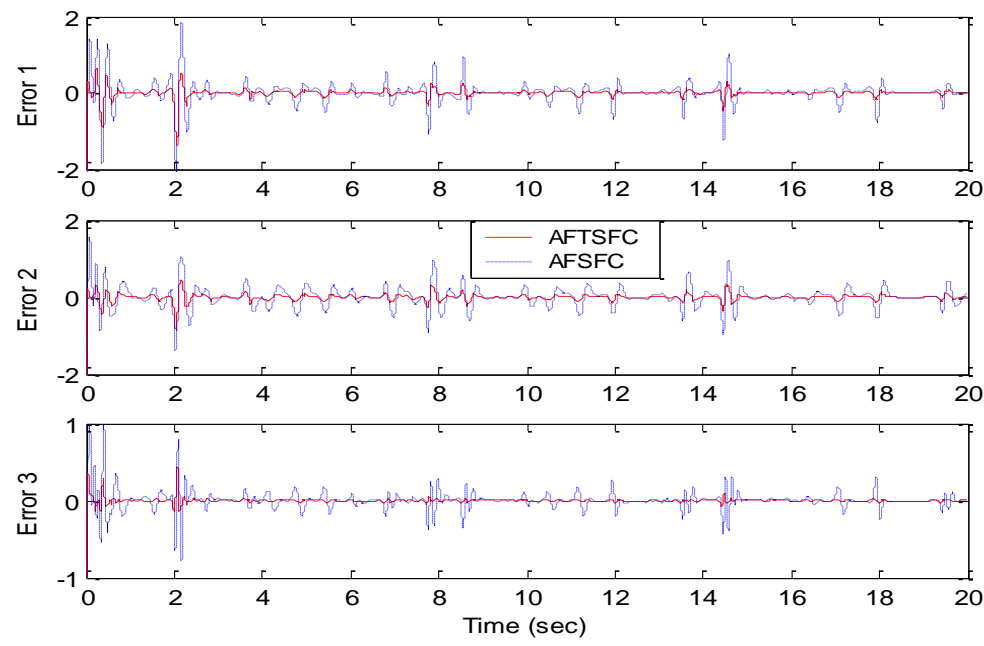

Figure 6(b). The tracking errors of the drive and response systems under the AFTSFC (solid line) and the AFSFC (dashdot line) 
gain can be obtained from $\bar{K}_{j}=\bar{M}_{j} Q^{-1}$. The matrix $Q$ is listed as follow:

$$
Q=\left[\begin{array}{cccccc}
0.2114 & -0.0304 & -0.0004 & -0.6287 & 0.0446 & 0.0008 \\
-0.0304 & 0.0544 & -0.0001 & 0.0337 & -0.2138 & -0.0009 \\
-0.0004 & -0.0001 & 0.0808 & 0.0005 & 0.0001 & -0.3278 \\
-0.6287 & 0.0337 & 0.0005 & 2.9645 & 0.4769 & 0.0077 \\
0.0446 & -0.2138 & 0.0001 & 0.4769 & 2.5755 & 0.0117 \\
0.0008 & -0.0009 & -0.3278 & 0.0077 & 0.0117 & 2.6895
\end{array}\right] .
$$

Using the adaptive law (25) and (23), we can obtain $k$ and $h$ at $t=20 \mathrm{sec}$ as follows:

$$
k=\left[\begin{array}{ccc}
34.9170 & 18.9624 & 0.7862 \\
13.1810 & 43.9672 & -1.8051 \\
0.5179 & -1.0966 & 10.6436
\end{array}\right], h=\left[\begin{array}{ccc}
38.2662 & 16.0026 & 0.4764 \\
13.5941 & 73.1674 & 0.2819 \\
0.3942 & 0.9418 & 56.1254
\end{array}\right] .
$$

The trajectories of the drive and response systems under the proposed AFTSFC are shown in Figure 6a. The tracking errors of the drive and response systems under these two controllers are shown in Figure 6b.

\subsection{Example 3}

In this example, a Rössler system and unified system are employed as the drive and response chaotic systems, respectively. The proposed approach is then employed to realize the synchronization. The Rössler drive system is described as equation (41), while the fuzzy unified response system is described as equation (49).

In this example, the TSF is defined as (43), and the FTSF is given as (44). The membership functions are shown in Figure 3. The fuzzy state-feedback controller is given as (46). The membership functions are shown in Figure 3. In the AFSFC, classical sliding function is defined as (47). The initial states of the drive and response systems are $\hat{\mathbf{x}}=\left[\begin{array}{lll}0 & 1 & 0\end{array}\right]^{T}$ and $\mathbf{x}=\left[\begin{array}{lll}-10 & -10 & -10\end{array}\right]^{T}$, respectively. In the drive system, we choose $\hat{a}=0.34+\frac{0.34}{2} \cos (t)$, $\hat{b}=0.4+\frac{0.4}{2} \cos (t), \hat{c}=\frac{\hat{c}_{2}+\hat{c}_{1}}{2}+\frac{\left(\hat{c}_{2}-\hat{c}_{1}\right)}{2} \cos (t), \hat{c}_{1}=4.5+\frac{4.5}{2} \cos (t), \hat{c}_{2}=7.7+\frac{7.7}{2} \cos (t)$, and in the response system, we choose $a=0.5$, and this is regarded as a parameter mismatch. By choosing $\gamma=10$ and $\rho=1, \bar{M}_{j}$ and $Q$ can be obtained from (40) using the MATLAB LMI toolbox. Then, the feedback gain can be obtained from $\bar{K}_{j}=\bar{M}_{j} Q^{-1}$. The matrix $Q$ is listed as follow:

$$
Q=\left[\begin{array}{cccccc}
0.2114 & -0.0304 & -0.0004 & -0.6287 & 0.0446 & 0.0008 \\
-0.0304 & 0.0544 & -0.0001 & 0.0337 & -0.2138 & -0.0009 \\
-0.0004 & -0.0001 & 0.0808 & 0.0005 & 0.0001 & -0.3278 \\
-0.6287 & 0.0337 & 0.0005 & 2.9645 & 0.4769 & 0.0077 \\
0.0446 & -0.2138 & 0.0001 & 0.4769 & 2.5755 & 0.0117 \\
0.0008 & -0.0009 & -0.3278 & 0.0077 & 0.0117 & 2.6895
\end{array}\right] .
$$

Using the adaptive law (25) and (23), we can obtain $k$ and $h$ at $t=100 \mathrm{sec}$ as follows: 


$$
k=\left[\begin{array}{ccc}
241.6422 & 7.5790 & 19.0857 \\
17.1752 & 105.5087 & -0.4741 \\
17.6141 & -1.4347 & 58.0472
\end{array}\right], h=\left[\begin{array}{ccc}
36.9122 & 15.0133 & 0.6964 \\
11.6987 & 71.2306 & 0.7384 \\
0.3164 & 0.7429 & 56.0396
\end{array}\right]
$$

The trajectories of the drive and response systems under the proposed method are shown in Figure $7 \mathrm{a}$. The tracking errors of the drive and response systems under these two controllers are shown in Figure 7b.
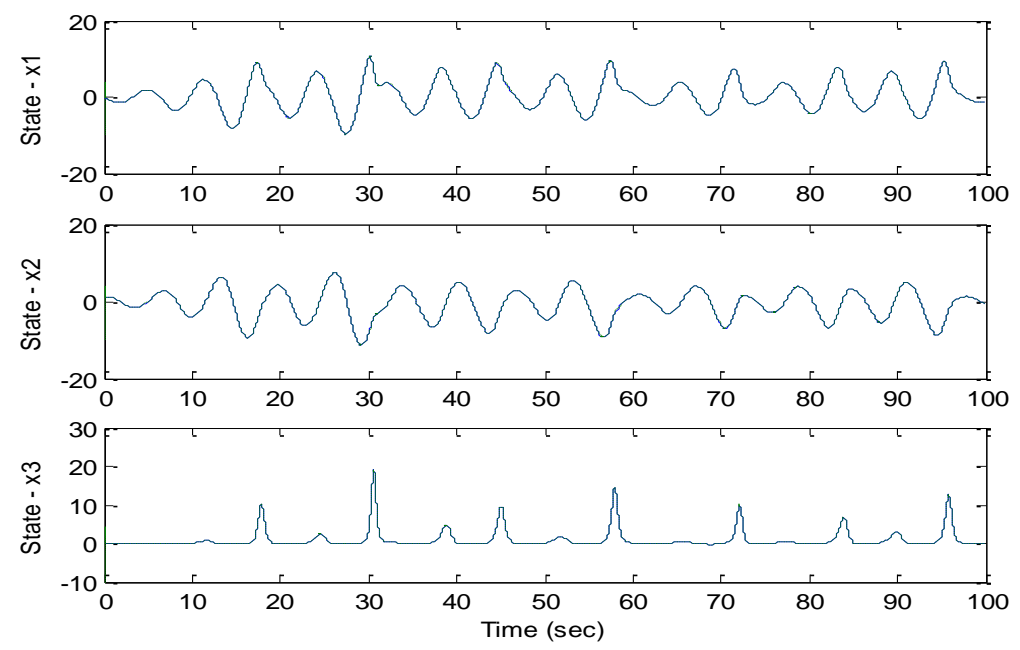

Figure 7(a). The trajectories of the drive (solid line) and response (dashdot line) system under the proposed AFTSFC

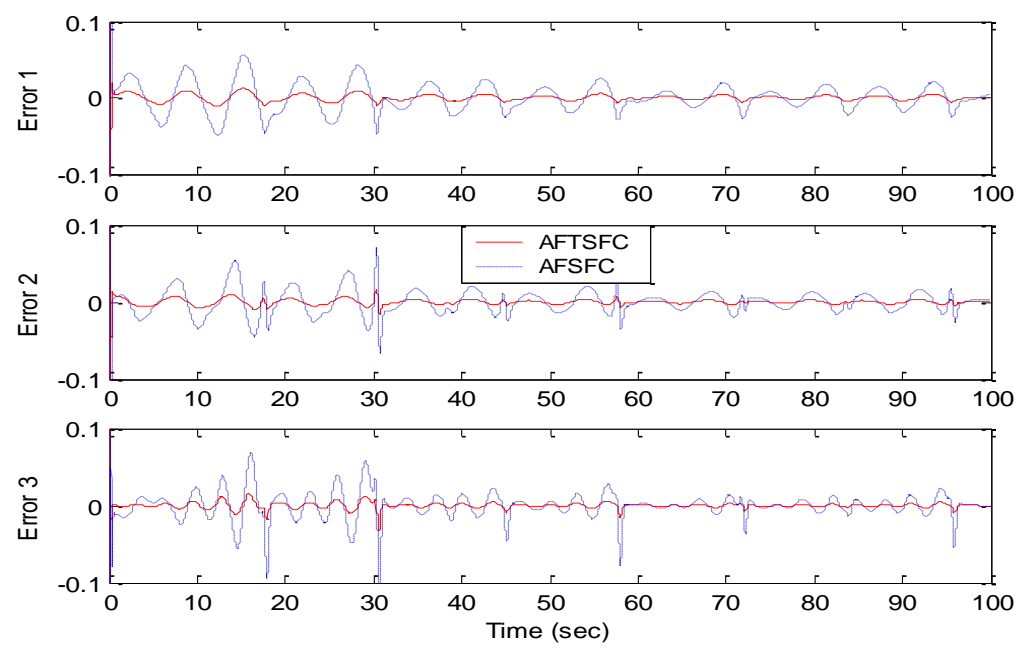

Figure 7(b). The tracking errors of the drive and response systems under the AFTSFC (solid line) and the AFSFC (dashdot line)

In all examples, the performance indices of the corresponding controller are tabulated in Table 1. By referring to Figures 5, 6, and 7, it can be seen that the proposed approach is able 
to drive the system states of the parameter mismatch response to follow those of the drive system with a sufficiently small tracking error. With a comparison to Figures. 5, 6, 7, and Table 1, one can conclude that the synchronization performance of the proposed AFTSFC is superior to that of the AFSFC.

Table 1. The performance indices

\begin{tabular}{cccc}
\hline Example & Index & AFTSFC & AFSFC \\
\hline $\begin{array}{c}\text { Example } 1 \\
\text { Rossler chaotic } \\
\text { system }\end{array}$ & IAE & 0.1044 & 0.6633 \\
\hline $\begin{array}{c}\text { Example } 2 \\
\text { Unified } \\
\text { chaotic system }\end{array}$ & ITAE & 2.9424 & 33.8469 \\
\hline $\begin{array}{c}\text { Example } 3 \\
\text { Two different chaotic } \\
\text { systems }\end{array}$ & IAE & 1.5460 & 5.6271 \\
\hline IAE $=\int_{0}^{t_{f}}|e(t)| d t$, & ITAE & 10.5731 & 42.8223 \\
\hline
\end{tabular}

\section{Conclusions}

In this paper, we have developed the AFTSFC for synchronization of parameter mismatch chaotic systems. The combined scheme is shown to have the merits of these approaches. This control algorithm is designed on the basis of the Lyapunov stability criterion, which can be applied to two chaotic systems subject to parameter mismatch and two different chaotic systems. The proposed method is shown to have the following two characteristics. First, the presented scheme can drive the system tracking error to become sufficiently small. Second, the adaptive scheme deals with the parameter uncertainty and parameter mismatch of the chaotic systems. The effectiveness and validity of the AFTSFC are demonstrated by computer simulations, and the proposed control can achieve a satisfactory synchronization performance.

There are some studies still to be done in the near future. First, a non-singular terminal sliding-mode controller and a non-singular terminal sliding-function controller can be developed for chaotic systems. Second, a discrete-time terminal sliding-function controller can also be developed. Final, the performance of the controller can be improved to achieve asymptotic convergence without chattering.

\section{References}

[1] H. K. Lam and L. D. Seneviratne, "Chaotic synchronization using sampled-data fuzzy controller based on fuzzy-model- based approach”, IEEE Circuits Syst. I, Reg. Papers, vol. 55, no. 3, (2008), pp. 883-892.

[2] M. Ababneh, A. M. Almanasreh and H. Amasha, "Design of digital controllers for uncertain chaotic systems using fuzzy logic", Journal of the Franklin Institute, vol. 346, no. 6, (2009), pp. 543-556.

[3] F.-H. Hsiao, "Fuzzy control of dithered chaotic systems via neural-network-based approach", Journal of the Franklin Institute, vol. 347, no. 7, (2010), pp. 1114-1136.

[4] M. A. Khanesar, O. Kaynak and M. Teshnehlab, "Observer-based indirect model reference fuzzy control system with application to control of chaotic systems", Journal of the Franklin Institute, vol. 350, no. 3, (2013), pp. 419-436.

[5] J. Dong, Y. Wang and G.-H. Yang, "Control synthesis of continuous-time T-S fuzzy systems with local nonlinear models”, IEEE Trans. Syst. Man. Cybern. B, Cybern., vol. 39, no. 5, (2009), pp. 1245-1258. 
[6] K. Tanaka, T. Ikeda and H. O. Wang, "A unified approach to controlling chaos via an LMI-based fuzzy control system design”, IEEE Trans. Circuits Syst. I, Fundam. Theory Appl., vol. 45, no. 10, (2009), pp. 1021-1040.

[7] H. K. Lam, W. K. Ling, H. C. Iu and S. H. Ling, "Synchronization of chaotic systems using time-delayed fuzzy state-feedback controller”, IEEE Trans. Circuits Syst. I, Reg. Papers, vol. 55, no. 3, (2008), pp. 893903.

[8] P. J. Guo, X. Z. Wei, K. S. Tang and G. Chen, "Integral-observer-based chaos synchronization", IEEE Trans. Circuits Syst. II, Exp. Briefs, vol. 53, no. 2, (2006), pp. 110-114.

[9] C.-C. Cheng, Y.-S. Lin and S.-W. Wu, "Design of adaptive sliding mode tracking controllers for chaotic synchronization and application to secure communications", Journal of the Franklin Institute, vol. 349, no. 8, (2012), pp. 2626-2649.

[10] C. Yin, S. Dadras and S. -M. Zhong, "Design an adaptive sliding mode controller for drive-response synchronization of two different uncertain fractional-order chaotic systems with fully unknown parameter", Journal of the Franklin Institute, vol. 349, no. 10, (2012), pp. 3078-3101.

[11] M. Y. Hsiao, T. -H. S. Li, J. Z. Lee, C. H. Chao and S. H. Tsai, "Design of interval type-2 fuzzy sliding-mode controller", Information Sciences, vol. 178, no. 15, (2008), pp. 1696-1716.

[12] J. Wang, D. Q. Guo and B. Deng, "Observer-based robust adaptive variable universe fuzzy control for chaotic system”, Chaos Solitons Fractals, vol. 23, no. 3, (2005), pp. 1013-1032.

[13] E. Cherrier, M. Boutayeb and J. Ragot, "Observers-based synchronization and input recovery for a class of nonlinear chaotic models", IEEE Trans. Circuits Syst. I, Reg. Papers, vol. 53, no. 9, (2006), pp. 1977-1988.

[14] J. Lu, J. Cao and D. W. C. Ho, "Adaptive stabilization and synchronization for chaotic Lur'e systems with time-varying delay”, IEEE Trans. Circuits Syst. I, Reg. Papers, vol. 55, no. 5, (2008), pp. 1347-1356.

[15] J. H. Kim, C. H. Hyun, E. Kim and M. Park, "Adaptive synchronization of uncertain chaotic systems based on T-S fuzzy model”, IEEE Trans. Fuzzy Syst., vol.15, no. 3, (2007), pp. 359-369.

[16] S. Boyd, L. E. Ghaoui, E. Feron, V. Balakrishnan, Linear Matrix Inequalities in System and Control Theory, SIAM, Philadelphia, PA (1994).

[17] F. Piltan, N. Sulaiman, S. Soltani, M. H. Marhaban and R. Ramli, "An Adaptive sliding surface slope adjustment in PD Sliding Mode Fuzzy Control for Robot Manipulator", International Journal of Control and Automation, vol. 4, no. 3, ( 2011), pp. 65-76.

[18] G. V. Lakhekar, "Tuning and Analysis of Sliding Mode Controller Based on Fuzzy Logic", International Journal of Control and Automation, vol. 5, no. 3, (2012), pp. 93-110.

[19] C. W. Tao, J. Taur, J. H. Chang and S. -F. Su, "Adaptive fuzzy switched swing-up and sliding control for the double-pendulum-and-cart system”, IEEE Trans. Syst. Man. Cybern. B, Cybern., vol. 40, no. 1, (2010), pp. 534-539.

[20] T. -H. S. Li and S. -H. Tsai, "T-S fuzzy bilinear model and fuzzy controller design for a class of nonlinear systems", IEEE Trans. Fuzzy Syst., vol. 15, no. 3, (2007), pp. 494-506.

[21] J. Dong, Y. Wang and G. -H. Yang, "Control synthesis of continuous-time T-S fuzzy systems with local nonlinear models", IEEE Trans. Syst. Man. Cybern. B, Cybern., vol. 39, no. 5, (2009), pp. 1245-1258.

[22] M. Zhihong, A. P. Paplinski and H. R. Wu, "A robust MIMO terminal sliding mode control scheme for rigid robotic manipulators", IEEE Trans. Autom. Control, vol. 39, no. 12, (1994), pp. 2464-2469.

[23] M. Zhihong and X. H. Yu, "Terminal sliding mode control of MIMO linear systems", IEEE Trans. Circ. Syst. I, Fundam. Theory Appl., vol. 44, no. 11, (1997), pp. 1065-1070.

[24] X. H. Yu and M. Zhihong, "Fast terminal sliding-mode control design for nonlinear dynamical systems", IEEE Trans. Circ. Sys. I, Fundam. Theory Appl., vol. 49, no. 2, (2002), pp. 261-264.

[25] T. -H. S. Li and Y. -C. Huang, "MIMO adaptive fuzzy terminal sliding-mode controller for robotic manipulators", Information Sciences, vol. 180, no. 23, (2010), pp. 4641-4660.

[26] T. -H. S. Li and Y. -C. Huang," An MIMO terminal sliding function controller design based on parameter estimation of the T-S fuzzy model approach", Int. J. Nonlinear Sci. Numer. Simul., vol. 11, (2010), pp. 103107.

[27] C. -C. Yang, "Synchronization of second-order chaotic systems via adaptive terminal sliding mode control with input nonlinearity", Journal of the Franklin Institute, vol. 349, no. 6, (2012), pp. 2019-2032. 
International Journal of Control and Automation Vol.7, No.2 (2014) 